\title{
Ludwig Winders Psychogramm und seine Bezugnahme auf die Psychoanalyse in Der Thronfolger und in Die nachgeholten Freuden
}

\section{Ludwig Winder's Psychogram and His Reference to Psychoanalysis in the novels Der Thronfolger and Die nachgeholten Freuden}

Wolfgang Müller-Funk

\begin{abstract}
Unlike other great novels on the decline and fall of the Habsburg Empire, such as Radetzkymarsch by Joseph Roth or Der Mann ohne Eigenschaften by Robert Musil, Winder's novels on this subject matter reveal a strong matriarchal determination of male protagonists which, however, is broken down in many ways. In Der Thronfolger (1937), the neurotic agility of the Austrian heir to the throne is actually an implant, or even a loan from female mother figures (including his wife) of the novel, which themselves have in turn adopted many compensational behaviour patterns originating from their fathers. Even stronger oedipal motives are exposed in the power-obsessed, capitalist siege of a small Bohemia town by Adam Dupic, a mysterious foreigner, in Winder's 1927 novel Die nachgeholten Freuden. Both novels can thus be read as a specific version of the 'Habsburg complex', displaying yet another aspect of the fragility of the modern condition in Central Europe.
\end{abstract}

\section{Keywords}

psychoanalysis; crisis of patriarchy; matriarchy; late Habsburg period; Oedipus complex; autoritarianism 
Der Erste Weltkrieg und die darauffolgende kurze Periode bis zum Untergang der demokratischen Staatsgebilde in Europa, der Triumph autoritärer, faschistischer, nationalsozialistischer und stalinistischer Regime hat die Psychoanalyse nachhaltig verändert. Schon vor dem Ersten Weltkrieg haben Freud und sein Umfeld den Versuch unternommen, die Psychoanalyse nicht nur als psychologische Theorie und als Therapie, sondern auch als eine Form der Kulturanalyse zu begreifen. Der Erste Weltkrieg und die daran anschließenden gesellschaftspolitischen Krisen haben die Entwicklung beschleunigt. Ich denke dabei an Freuds unmittelbar nach dem Ersten Weltkrieg entstandene Studie Jenseits des Lustprinzips, seine Massenpsychologie oder die 1930 - als sich der Untergang der Weimarer Republik in Deutschland abzuzeichnen beginnt - erschienene Schrift Das Unbehagen in der Kultur.

Die Echos des von Freud initiierten Diskurses hallen in vielen Werken der österreichischen Moderne zustimmend wie ablehnend nach, bei Robert Musil, Hermann Broch, Elias Canetti, Stefan Zweig, Ödön von Horváth, Franz Kafka, Ernst Weiß oder auch bei Ludwig Winder. Wie intensiv die genannten Autoren sich mit Freuds Psychoanalyse beschäftigt haben, wäre Gegenstand einer aufwendigen, aber lohnenswerten Studie; es könnte sich hie und da auch um Parallelaktionen handeln. Oder anders ausgedrückt: Die Krise der symbolischen Ordnung, die Infragestellung der patriarchalen Gesellschaft, der Ödipus-Konflikt und die Beschäftigung mit der Figur der Mutter sind kulturelle Zeiterscheinungen, auf die die Psychologie als neue wissenschaftliche Disziplin ebenso reagiert wie die Autoren der mittlerweile klassischen Moderne; dazu zählen von deutscher Seite gewiss auch die Gebrüder Heinrich und Thomas Mann oder Lion Feuchtwanger, deren Romane spürbar psychoanalytisch amalgamiert sind.

Franz Ferdinand, der Thronfolger in Ludwig Winders gleichnamigem Roman erweist sich als eine schwache Figur insbesondere in der Auseinandersetzung mit dem Langzeit-Kaiser Franz Joseph. Es gelingt ihm nicht, sich von der geliebten Mutter und den nachfolgenden Muttergestalten zu lösen und positiv die Vaterrolle zu besetzen. Das wäre Freud zufolge der produktive Ausgang aus dem Ödipus-Konflikt: Loslösung vom Begehren, die Mutter für sich zu besitzen, und die Überwindung des Wunsches, den Vater als Rivalen zu beseitigen. Aber zugleich wird das politische Geschehen in diesem Sinne gedeutet: Denn der Untergang der alten Imperien in Europa ist kulturell betrachtet ein Symptom der Krise der symbolischen Ordnung, die auf dem Gesetz des Vaters beruht.

Winders umfangreicher Text firmiert als Roman; genauer als historischer Roman, doch im Gegensatz zu den Hauptströmungen dieser Sub-Gattung - ich denke im deutschsprachigen Kontext etwa an Lion Feuchtwangers historische Romane oder an Heinrich Manns Opus magnum Henri Quatre - folgt der Autor getreu den historischen Fakten. Was ,Fiktion“ ist, das sind über die Fabelkonstruktion und die damit einhergehenden Interpretationen hinaus die Fokalisierung sowie die erfundenen Dialoge, die mit den psychologischen Deutungen der Person Hand in Hand gehen und die Innenwelt eines Menschen freilegen, der von Selbstzweifeln, von einer dominanten Mutter und von Introvertiertheit bestimmt ist. Die Fabelkonstruktion lässt keinen Zweifel daran, dass der linksrepublikanische Verfasser keinerlei Sympathie für das Haus Habsburg und den Patriarchalismus des ancièn regime hegt. Die beinahe psychoanalytische Beleuchtung 
der Figur reduziert Franz Ferdinand auf eine menschliche Größe, aber sie vernichtet ihn bemerkenswerterweise nicht, sondern zeigt den Thronfolger vielmehr in einer zwiespältigen Situation. Zum einen ist der Kaiserneffe Teil eines im Roman als überholt angesehenen Herrschaftskomplexes, zum anderen aber, die Herrschaftskritik bestätigend, wird er, der von der kaiserlichen Familie wegen seiner ,unstandesgemäßen` Ehe gedemütigte Mann, bis zu einem gewissen Grade auch deren Opfer. Seine prekäre Stellung in der Herrscherfamilie führt letztendlich zu einem Verhängnis, das weit mehr ist als das seine, zum Tod in Sarajevo. Er zeigt Stärke aus Gegenwehr gegen die Herkunftsfamilie und will auf keinen Fall ein Versager sein.

Diesen beiden Umständen, äußerer Faktentreue ${ }^{1}$ und dem psychoanalytisch-psychologischen Profil verdankt Winders Roman, dass er, wie Ulrich Weinzierl bemerkt, bis zum heutigen Tage in einschlägigen historischen Standardwerken wie jenen von Friedrich Weissensteiner (2007) und Gordon Brook-Shepherd (1988) Erwähnung findet. ${ }^{2}$ So überschreitet das Werk die festgefügte traditionelle Grenze zwischen Literatur und Historiographie und provoziert zugleich die Frage, was Literatur, vielleicht auch im Unterschied zur Historiographie, zur Erhellung historischer Ereignisse beizutragen vermag.

Der sozialpsychologisch angelegte Text setzt an einem scheinbaren Nebenschauplatz der europäischen Geschichte ein, mit König Ferdinand II., dem „König beider Sizilien“, der von seinen Untertanen den Spottnamen ,Re Bomba' erhalten hat, weil er ganz offenkundig und ungeniert sein bedrohtes Reich autokratisch regiert und mit Brutalität und Gewalt zusammenzuhalten trachtet. 1859, in einem Schicksalsjahr für das österreichische Kaisertum, fällt der Großvater des späteren Thronfolgers den Folgen eines Anschlags zum Opfer, 1861 verliert sein unfähiger Sohn das Königreich, das in den neuen Nationalstaat Italien eingegliedert wird. ${ }^{3}$

So etabliert Winders Roman eine Welt von Spiegelungen und Wiederholungen. Das Attentat von 1859 antizipiert das Attentat von Sarajevo, das autoritäre Regime von Ferdinand II. ähnelt dem Chronisten zufolge (vielleicht ist dieses Urteil zu streng) dem des franzisko-josephinischen Kaiserstaates. Stabil und sich in diesen patriarchalischen Systemen wiederholend ist indes ein dem Patriarchat zunächst entgegengesetzter Zug: die Dominanz der Frauen über ihre Männer und Söhne. Dieses Matriarchat im Patriarchat steht in auffälligem Gegensatz zu anderen literarischen Arbeiten am Habsburgischen Mythos: In Roths Radetzkymarsch wie in Musils Der Mann ohne Eigenschaften bleibt die Stelle der Mutter leer und unbesetzt. Die Frauen der Trottas verschwinden geräuschlos aus den literarischen Welten Roths. Musils Ulrich hat einen Vater mit Eigenschaften, der der Gentry der Monarchie angehört und seine Karriere seiner Loyalität gegenüber der Monarchie verdankt, aber die Beschaffenheit der Mutter bleibt, vorsichtig formuliert, blass und unbestimmt. Elle n'existe pas. ${ }^{4}$

1 Weinzierl, Ulrich (2014): Nachwort. In: Winder, Ludwig: Der Thronfolger. Wien: Zsolnay, S. 561-563.

2 vgl. Weinzierl [Anm. 1], S. 560, 563 (Sekundärliteratur zu Winder).

3 vgl. Winder, Ludwig (2014): Der Thronfolger. Ein Franz-Ferdinand-Roman. Wien: Zsolnay, S. 7-23.

4 Musil, Robert (1978): Der Mann ohne Eigenschaften. Reinbek: Rowohlt, S. 490. Hier das klassische Musil-Zitat aus dem Munde des Romanprotagonisten Ulrich: „Mich hat doch eine Mutter geboren und kein Tintenfass!“ Trotz dieser Beteuerung gegenüber der zeitweiligen Geliebten Gerda Fischel bleibt die Mutter 
Winders habsburgischer ,Komplex“ beruht demgegenüber darauf, dass es die ,herrscherlichen' Frauen sind, die den Fortbestand des patriarchalen Systems garantieren. Da ist zunächst Erzherzogin Sophie, die Mutter von Kaiser Franz Joseph und von Erzherzog Karl Ludwig, dem bedeutungslosen Bruder des Kaisers, dessen Ehen sie arrangiert. Dann sind da, im Kontext des Romans noch gewichtiger, die beiden Mütter des Thronfolgers, die leibliche Mutter Maria Annunciata, die Tochter des ,Bombenkönigs', und, nach deren frühzeitigem Ableben, Maria Theresia von Portugal, die mühelos in die Rolle der ehrgeizigen Mutter schlüpft. Die nur acht Jahre ältere Stiefmutter wird für lange Zeit zu seiner bestimmenden Bezugsperson, der seine ganzen Zärtlichkeitsausbrüche gelten. Zur Familienaufstellung gehört auch, dass der jüngere Bruder, der schöne Otto, vom Vater und von seiner Umgebung bevorzugt wird. In dieser Situation beschließt die junge Stiefmutter, den verschlossenen und unsicheren Buben an sich zu binden:

„Sie war nicht oder noch nicht imstande, den zehnjährigen mürrischen, launenhaften, düsteren Stiefsohn zu lieben; sie belog deshalb sich und ihn - sie glaubte, es sei ihre Pflicht. Sie sagte ihm, er sei ihr näher, herzensnäher als die andern; aber er dürfe es keinem sagen. Eine heiße Welle von heißer Dankbarkeit schlug ihr entgegen. Sie sah Franz Ferdinands Augen aufleuchten, strahlen. Aber sie sah auch das hastige Zurückfluten dieses Gefühls, das Erwachen des Misstrauens, das seinen impulsiven Zärtlichkeitsausbrüchen folgte. Sie erriet seine Gedanken. Er dachte: Ist es möglich, dass mich jemand lieber hat als ihn [seinen Bruder Otto, A. d. V.]? Warum sollte sie mich lieber haben?

[...]

Trotzdem wuchs seine Zuneigung zu der jungen Frau, die er Mutter nannte. Er rief sie: ,Mama‘ Er nannte sie in seinen Gedanken: Mutter. Das war schon eine Erleichterung, eine Aufhellung seines Lebens. Er suchte ihre Augen, die ihn gütig anblickten, öfter als die gütigen, aber gleichgültigen Augen des Vaters. Er verzieh dem Vater nicht die Bevorzugung des jüngeren Bruders, weil er sie begriff." ${ }^{5}$

Aus dieser vertrackten und unauflöslichen ödipalen Konstellation, die zu keinem positiven Bild seines männlichen Selbst im Sinne Freuds führen wird, erklärt der Text auch Franz Ferdinands Unvermögen, soziale Beziehungen einzugehen, mit Männern wie mit Frauen. Erst mit Gräfin Sophie von Chotek von Chotkow und Wognin, der dritten starken Frau des späteren Thronfolgers, findet er im Ansatz jene Form von Anerkennung, die es ihm gestattet, selbst in die Rolle des Mannes und Vaters einzutreten. Aber der Preis dafür ist abermals, dass die neue soziale und emotionale Kompetenz gleichsam von

Ulrichs eine Fußnote, während der Vater, dessen Lebenszeit fast kongruent mit der Regierungszeit des Kaisers ist, in mehreren Kapiteln einen prominenten Auftritt bekommt. Dass die Familienkonstellationen des Romanprotagonisten und des ,realen“ Autors differieren, ist offenkundig. Denn dass Musils Mutter die dominante Figur in der Familie gewesen ist, ist wohl unbestreitbar - Spuren dieser Hegemonie finden sich etwa in Tonka, aber auch in Die Verwirrungen des Zöglings Törleß, vgl. dazu: Wolf, Norbert Christian (2011): Kakanien als Gesellschaftskonstruktion. Robert Musils Sozioanalyse des 20. Jahrhunderts. Wien: Böhlau, S. 1159; Pfohlmann, Oliver (2016): Biografie. In: Nübel, Birgit - Wolf, Norbert Christian (hrsg.): Robert Musil Handbuch. Berlin - Boston: de Gruyter, S. 1-4.

$5 \quad$ Winder [Anm. 3], S. 69. 
der Frau geliehen ist. Denn Sophie tritt nun als Ehefrau und Geliebte in die Position der dominanten Frau ein, die zuvor die beiden Mütter eingenommen haben. Von Anfang an umsorgt, beschützt und beherrscht sie ihn emotional. Schon bei ihrem ersten Aufeinandertreffen ringt sie ihrem neuen, gänzlich unerwarteten Verehrer das Versprechen ab, seine schwächliche Konstitution, Symptom seines schwachen männlichen Selbstbewusstseins, auszukurieren:

\begin{abstract}
„Die schönen braunen Augen baten. Es war in ihnen eine beschwörende Kraft. ,Sie hypnotisieren mich, Gräfin', lächelte er; ,wenn sie mich so anschaun, kann ich nicht nein sagen. ' - ,Also: Sie haben es mir versprochen, Kaiserliche Hoheit', sagte sie so ernst, so dringend, dass er nicht mehr lächeln konnte. Er sagte: ,Schön, ich verspreche. Aber nur Ihnen zuliebe. - ,Ich danke, Kaiserliche Hoheit', flüsterte sie so leise, dass er es kaum hörte. Aber deutlich las er Hingebung und eine unsagbare Zärtlichkeit in ihren Augen“. ${ }^{6}$
\end{abstract}

Literarisch bedient sich Winders ,middle brow'-Roman, der Literatur und Journalismus auf eine damals durchaus neue Art und Weise miteinander verknüpft, moderner, wenn auch nicht avancierter literarischer Techniken. Im Roman wechselt die Fokalisierung; die erlebte Rede gestattet es überdies, die Erzähler- mit der jeweiligen Figurenrede zu verbinden. Dadurch wird komplementär zur Welt der äußeren Ereignisse eine Binnenwelt geschaffen, die keineswegs auf den Thronfolger beschränkt ist, sondern in die vielmehr auch seine dominanten Bezugspersonen, die beiden Mütter und die Ehefrau, einbezogen sind. Daraus entsteht ein Geflecht von Relationen, das über das Persönliche hinaus das politische Handeln des Thronfolgers bestimmt. Die Entscheidung, zu den kaiserlichen Manövern in Bosnien zu fahren und diese zu leiten, ist hierfür paradigmatisch. Treibendes Motiv dabei, so das Narrativ in Winders Thronfolger, ist, zusammen mit seiner Frau als zukünftiger Kaiser Anerkennung zu erlangen und damit die Demütigungen, denen seine nicht standesgemäße, weil nicht dem europäischen Hochadel angehörende Frau seit ihrer Eheschließung ausgesetzt ist, wettzumachen. Die Reise nach Sarajevo bedeutet demgemäß auch, seine Frau als gleichberechtigte Gemahlin des Thronfolgers zu zeigen. Es sind die „mit Hochmut und Schadenfreude“ verbundenen „Demütigungen“, die letztendlich zur Motivation für den Besuch des 1908 annektierten Protektorats Bosnien-Herzegowina werden. ${ }^{7}$

Winders Studie des Langzeit-Kronprinzen verschiebt den narrativen Komplex der späten, also franzisko-josephinischen Habsburger Monarchie auf eine erstaunliche und überraschende Weise. Die Identitätskrise der Männlichkeit ist dabei auf zwei Ebenen angesiedelt, auf einer privaten wie auf einer politischen. Roths Niedergangserzählung im Radetzkymarsch sieht die Ursache der Krise höchst konservativ darin, dass insbesondere der Enkel nicht mehr dem großen, positiven Bild des Großvaters, des Helden von Solferino, zu genügen vermochte. Musils Ulrich verschmäht es hingegen, in die symbolische Ordnung des Vaters einzutreten. Winders männlichem Protagonisten - weder ein Rebell

$6 \quad$ Winder [Anm. 3], S. 209.

7 Winder [Anm. 3], S. 490. 
noch ein ironischer Außenseiter - gelingt dieser heiß ersehnte Schritt nur in einem sehr zwiespältigen und gebrochenen Modus. Zum einen widersetzt er sich ihr mit seiner in den Augen des Kaiser-Vaters nicht standesgemäßen Heirat und zum anderen gründet seine fragile männliche Identität auf einer starken und einseitigen Abhängigkeit von den Frauen; diese prekäre Relation vermag er nicht positiv in einem modernen, etwa emanzipatorischen Sinn zu wenden. Die letzten Jahrzehnte der habsburgischen Herrschaft beruhen demnach auf einer klandestinen und indirekten Machtposition von Frauen, die die alte Ordnung auf ihre Weise zu retten trachten.

Winder deutet Franz Ferdinands kränkliche Konstitution und schwächliche Männlichkeit als Symptom einer generellen Krise der patriarchalen Ordnung. Der furchtbare Jagdeifer des Kronprinzen, der an einem Tag fünfzig Gamsböcke erlegt, wird im Sinne einer männlichen Ersatzhandlung und als Ausdruck eines gestörten Verhältnisses zu seiner Umwelt gedeutet, das er, so die Erzählerrede, systematisch verkennt:

„Franz Ferdinand ging auf die Jagd. Er schoss an einem Tag dreiundfünfzig Gamsböcke. Die Treiber zitterten, wenn er sie anblickte. Auch sie fürchteten ihn, auch sie fluchten ihm.

Er wusste es nicht. Er wusste nicht, dass er die Menschen verachtete. Es gab nur einen Menschen, den er liebte: Sophie. Einen, den er verehrte: die Stiefmutter [...] Einen, den er fürchtete: den Kaiser."8

Kein Zufall übrigens, dass die meisten dieser selbstbewussten Frauen selbst Verlust und Marginalisierung erfahren haben, die zwei Mütter des Thronfolgers, deren Väter beide des Throns verlustig gegangen waren, ebenso wie seine spätere Frau, deren Vater als Statthalter von Böhmen auch schon bessere Tage erlebt hatte. Es sind diese Erfahrungen, die das Handeln der Frauen um den Thronfolger bestimmen. Als sich am 30 . Jänner 1889 die Nachricht vom Suizid Erzherzog Rudolphs verbreitet, ist es die nur um acht Jahre ältere geliebte Stiefmutter, die die Initiative ergreift, wenn sie ihm unmissverständlich erklärt:

„,Mach Dir keine Sorgen, und sei tapfer, Franzi, du wirst deine Kräfte brauchen. Ist dir auch klar, wie ungeheuer sich gestern dein Schicksal geändert hat? Die ganze Welt sieht in dir den künftigen Kaiser. Dass Papa offiziell nicht verzichtet, ist bedeutungslos, denn er ist bereit, dir rechtzeitig den Platz zu räumen. Der wirkliche Thronfolger bist du, wenn es auch offiziell nicht ausgesprochen wird. Man wird viel von dir verlangen. Du musst deshalb kräftiger werden und gesund bleiben, das ist die Voraussetzung. Also schon dich, und bereite dich ohne Hast und ohne Ungeduld auf dein hohes Amt vor. Ich hoffe und glaube, dass du zu Großem berufen bist. Warte geduldig deine Stunde ab! Sei nicht unglücklich und glaub an dich! Ich will dich glücklich wissen; lieber glücklich als ruhmreich und groß.‘

Dankbar umarmte er sie. Sie küsste ihn errötend und verließ das Zimmer. Er ging zu Bett.“9

8 Winder [Anm. 3], S. 290.

9 Winder [Anm. 3], S. 147-148. 
Wenden wir im folgenden Gedankenexperiment die Freud'sche Orthodoxie auf Winders Franz Ferdinand an, so ergibt sich folgendes, durchaus erhellendes psychohistorisches Gesamtbild des habsburgischen ,Komplexes`, genauer des ungelösten kollektiven Ödipuskomplexes. Franz Ferdinand ist ganz offenkundig nicht imstande, in die Normalität der patriarchalen Ordnung des Vaters einzutreten und sich mit dem Ich-Ideal des Vaters, des leiblichen wie des symbolischen, des Kaisers und Onkels, zu identifizieren. In gewisser Hinsicht hält er an seiner Identifizierung mit der Mutter, hier der Stiefmutter, fest. Umgekehrt verweigern aber auch die dominierenden weiblichen Gestalten in gewisser Weise die Identifikation mit der Mutter und deren Rolle. Oder anders ausgedrückt: Sie nutzen sie zu ,männlichem‘ Tun. Beide Mütter des Thronfolgers identifizieren sich mit eben jenem Vater, der hier wie dort seinen Thron, Inbegriff seiner Männlichkeit, verloren hat. Sie setzen alles daran, dem Sohn in einem kompensatorischen Akt eine führende Stellung zu verschaffen bzw. für ihn schließlich gar den männlichen ,Thron` zu gewinnen und diesen zugleich historisch zu retten. Den Verlust der väterlichen Krone versuchen sie dadurch wettzumachen, dass sie eine noch mächtigere für den Sohn erringen. Aus diesen Überkreuzungen resultiert eine komplexe bisexuelle Konstellation. In Das Ich und das Es konstatiert Sigmund Freud 1923, ein gutes Jahrzehnt vor dem Erscheinen von Winders Der Thronfolger:

\begin{abstract}
„Der Ausgang der Ödipussituation in Vater- oder in Mutteridentifikation scheint also bei beiden Geschlechtern von der relativen Stärke der beiden Geschlechtsanlagen abzuhängen. Dies ist die Art, wie sich die Bisexualität in die Schicksale des Ödipuskomplexes einmengt. Die andere ist noch bedeutsamer. Man gewinnt nämlich den Eindruck, dass der einfache Ödipuskomplex überhaupt nicht das häufigste ist, sondern einer Vereinfachung oder Schematisierung entspricht $[\ldots]^{“} \cdot{ }^{10}$
\end{abstract}

Der ,vollständigere ‘ Ödipuskomplex besteht demzufolge darin, dass beide Geschlechter auf Vater und Mutter sowohl männlich wie auch weiblich zu reagieren vermögen. Die Familienaufstellung, die Winder in seinem ,Roman'vorführt, entspricht augenscheinlich nicht dem einfachen, sondern dem komplizierteren und ,vollständigeren“ Komplex. Ob die Protagonistinnen und Protagonisten im Thronfolger diesen Komplex tatsächlich überwinden, ist mehr als fraglich. Viel näher liegt der Befund, dass die in Winders Buch über Kronprinz Franz Ferdinand beschriebenen Konstellationen in höchstem Maße neurotisch sind, wobei die privaten auf die politischen umschlagen:

„Er hatte den Ehrgeiz, ein großer Herrscher zu werden und die Völker des großen Reichs zufriedenzustellen. Aber da er über die Menschen hinwegsah, kannte er sie nicht und erkannte nicht, dass er nur einen unversöhnlichen Feind hatte, der ihn unablässig quälte, narrte, um jede frohe Stunde, um jeden Erfolg und um jedes Glück betrog. Dieser Feind war er selbst.“11

10 Freud, Sigmund (1992): Das Ich und das Es. Metapsychologische Schriften. Einleitung von Alex Holder. Frankfurt a. M.: Fischer, S. 272.

11 Winder [Anm. 3], S. 291. 
Ludwig Winders Roman Die nachgeholten Freuden kreist wiederum um das ödipale Dreieck. Mit der Figur des sozialen Aufsteigers Adam Dupic verbindet der Roman diese soziale Charaktermaske des Kapitals mit einem Psychogramm, das dem Freud'schen, teils auch dem Canetti'schen Diskurs entnommen sein könnte. Bereits der Titel referiert auf einen freudianischen Diskurs, denn die nachgeholten Freuden verweisen auf eine verschobene Triebstruktur, in der die traumatisch verpasste kindliche Libido durch die Freude an einer Macht kompensiert wird, deren autodestruktive Dimension am Ende des Romans zutage tritt. Am Ende beharrt er darauf im Bett der Mutter zu sterben - eine regressive Selbstauflösung ist da im Spiel, die vor allem im theoretischen Werk von Sándor Ferenczi eine maßgebliche Rolle spielt, hat doch der viele Jahre engste Weggefährte Freuds die geschlechtliche Vereinigung im Sinn des männlichen Wunsches gedeutet, in den mütterlichen Schoß zurückzukehren.

Das ödipale Phantasma ist nicht zu übersehen. Da ist zunächst einmal die Schande der Mutter: Die Mutter ist die Hetäre schlechthin, der Pfuhl für alle Männer, die sie und ihren Körper genießen wollen, das Unreine, Grenzenlose, das jeden und alles in sich aufnimmt, so wie es etwa Bachofen in seinem Mutterrecht beschrieben hat. ${ }^{12}$ Der Vater hingegen ist impotent angesichts dieses magischen weiblichen Pfuhles. Verweigerung und Unfähigkeit sind hierbei zwei Seiten ein und derselben Medaille. Die Schamlosigkeit der mythischen Mutter und die Kastration des Vaters stellen eine doppelte, miteinander verquickte Schande dar, die nur durch Forcierung und Überbietung paternalistischer Geltungsansprüche zu durchbrechen ist. Der zukünftige Machthaber von Boran begibt sich auf die Flucht vor der Schande und den „Freuden“ der Mutter: Niemals will und wird er ihnen unterliegen. Indem er sich aktiv dem Pfuhl des Weiblichen entzieht, erwirbt er jene Selbstkontrolle, die Voraussetzung für die Macht über andere ist. Und doch bleibt das ambivalent, wird doch das Begehren (nach) der Mutter niemals wirklich gebrochen. Aus der Erfahrung extremer Ohnmacht, wie sie die Schande der Mutter, insbesondere aber die Impotenz des Vaters impliziert, erwächst der Wunsch, nie wieder ohnmächtig zu sein.

Dupic vergisst nicht, er verdrängt nicht im Sinne Freuds: Er führt das Inventar, das Indiz der Schande seiner Eltern immer mit sich, wie er der jungen Elsa, dem einzigen Menschen, dem er eine gewisse Achtung entgegenbringt, verrät:

„In diesem Bett hat mein Vater geschlafen. In diesem Bett hat meine Mutter geschlafen mit ihren Liebhabern. Man soll nicht trachten, seine Jugend zu vergessen, das kann man nämlich ohnehin nicht. Man soll sich's gemütlich machen auf dem Grab seiner Jugend, dann geht's einem gut. In keinem Bett kann ich so gut schlafen wie in dem Bett meiner Mutter."13

12 Bachofen, Johann Jakob (1984): Mutterrecht und Urreligion (6. erweiterte Ausgabe). Stuttgart: Kröner, z. B. S. 166 :

„Um das Weib dauernd zu besitzen, muß es der Mann erst andern überlassen. Nach dem ius naturale ist die Frau buhlerischer Natur, eine Acca Larentia, die ,dem Zufälligen` sich hingibt wie der Erdstoff, der als Penia nach immer erneuter Befruchtung sich sehnt. Das Weib, soll [...] bis zur Ermüdung dem Manne sich hingeben, wie Hortas Tempel bei den Römern immer offen stand.“

13 Winder, Ludwig (1987): Die nachgeholten Freuden [1927]. Wien: Zsolnay, S. 109. 
Im Gegensatz zur Mutter ist Elsa ,rein' ${ }^{14}$ und jungfräulich, sie verkörpert somit die andere Seite des männlichen Phantasmas der Frau. Vor allem aber ist Elsa nicht bereit, sich dem neuen Machthaber zu unterwerfen. Deshalb avanciert Elsa auch im Verlauf des Romans zur gefährlichsten Widersacherin des Schadenstifters Dupic.

Worin bestehen aber eigentlich Dupics nachgeholte Freuden? Nachgeholt werden zum einen die Freuden der Mutter, freilich asexuell, als sinn- und zwecklose Ausübung einer sich selbst frönenden Lust an der Macht. Diese Lust, die stärker ist als der schmutzige Sex, wird zum anderen nachgeholt, weil die Überwältigung der Menschen in der Heimat, der Großbauern, missriet. Schrankenlose Dominanz über die Anderen hat Fremdheit und Intransparenz zur Voraussetzung. Der Lustgewinn ist wiederum an die Ablehnung der durch die Mutter bezeichneten Freuden geknüpft. Weil Dupic Elsa begehrt (und damit zum Konkurrenten des Sohnes wird) - auch das ist eine ödipale Konstellation -, gefährdet diese seine Macht. Wenn unumschränkte Herrschaft nur in der Gestalt des Fremden (was natürlich auch eine Projektion der Beherrschten darstellt) möglich ist, dann bedeutet das auch, dass der Macht-Lustgewinn an den Verlust von Heimat gebunden ist.

Das ödipale Geschehen tritt auch darin sichtbar hervor, dass es die Erinnerung an die Kindheit ist, die Dupic am Ende buchstäblich und metaphorisch zu Fall bringt. Der kleine Sohn der Witwe, deren verschuldeter Mann Selbstmord begangen hat und Dupic = Dieb schreit, erinnert ihn an eine Begebenheit aus der eigenen Kindheit: Die Stimme holt seine Vergangenheit ein. In seinem Heimatort Dugosela versucht er nach dem Tod des Vaters, gleichsam die Herrschaft an sich zu reißen. Doch das Misstrauen der Großbauern gegen den reichen Dupic ist stärker. Und es ertönt eine Stimme, die seinen Namen lautmalerisch mit seinem Tun verquickt: Dupic $=$ Dieb. Die Stimme, Mimesis der Stimme Gottes, spricht das Urteil. Zum Schluss bittet er darum, im Bett, im sündigen Pfuhl der Mutter, zu sterben.

Die Anatomie der Macht enthüllt ein verqueres Verhältnis von Askese und Begehren: Dupics nachgeholte Freuden an der Macht verdanken sich nicht bloß der Abstinenz gegenüber dem schmutzigen, weiblichen Pfuhl (letztendlich gegenüber allen sinnlichen Freuden), sondern auch gegenüber dem Medium der eigenen Macht, dem Geld. Seine Askese ist nicht weniger radikal als die eines Mönchs, der freilich weiß, dass die Unterlage seines Tuns das Bett der Mutter ist. Sein einziges Begehren ist - ganz im Sinne Hegels - die Macht über den/die Anderen, mehr noch: „die Macht über dies Sein“. ${ }^{15}$ Im Kampfe mit den Anderen hat er bewiesen, dass ihm das Sein nur als Negatives gilt. Er sieht das Andere nicht als Wesen, sondern sich selbst im Anderen. Dieses Selbstbewusstsein macht das aus, was die Lacan-Schule als ,jouissance“ bezeichnet. ${ }^{16}$

14 Zum Phantasma der Reinheit vgl. Bauman, Zygmunt (1999): Das Unbehagen in der Postmoderne. Aus dem Englischen von Wiebke Schmaltz. Hamburg: Hamburger Ed., S. 14-34.

15 Hegel, Georg Wilhelm Friedrich (1970): Phänomenologie des Geistes. Werkausgabe, hrsg. von Eva Moldenhauer und Karl Markus Michel. Bd. 3. Frankfurt a. M.: Suhrkamp, S. 151.

16 vgl. Hegel [Anm. 15], S. 145-155. 


\section{Vollständige Unterwerfung und Demütigung}

„Ich kaufe zwar das Schloß, aber ich nehme es Ihnen nicht. Sie können es nach dem Verkauf weiterbewohnen wie bisher. Völlig unverändert. Ich verlange nur, daß Sie sich noch heute prinzipiell einverstanden erklären."17

Zur vollständigen Unterwerfung gehört, dem anderen keine Zeit zu lassen, ihn dem eigenen Zeitregime zu unterwerfen. Die kapitalistische Ökonomie funktioniert nach dem Gebot, dass Zeit Geld bedeutet und Zeitverlust überhaupt zu vermeiden ist. Dupic instrumentalisiert diese Eigenschaft moderner kapitalistischer Ökonomie für seinen Lustgewinn, indem er ein vorgeblich großzügiges Angebot mit der sofortigen Kapitulation des/der Anderen verknüpft. Das Primat des Soforts schafft tabula rasa und gilt uneingeschränkt. Das betrifft sein Vorgehen gegenüber der eigenen Familie wie gegenüber den adligen Besitzern von Schloss Boran. Mit dieser Vorgehensweise verschafft er sich absolute Handlungsfreiheit. Er verspricht den Geschwistern wie der verachteten Mutter eine Stange Geld unter der Voraussetzung, dass sie sofort, hic et nunc das eigene Haus verlassen. Er gibt - scheinbar großzügig - später den Bewohnern von Boran sogar zinslose Darlehen, verlangt aber dann sofort, am vereinbarten Tag, die (unmögliche) Rückzahlung. Er bringt die Menschen, die er verachtet, zu Fall, weil er um ihre Lüste und Schwächen, um ihre petits jouissances, weiß.

Macht ist mit Einsamkeit bezahlt. Dadurch wird auch scheinbar die Paradoxie auflösbar: Wie kann jemand die Menschen so abgrundtief verachten, wenn er selbst einer ist? Die unumschränkte Macht gestattet es, sich herauszuheben, Gottähnlichkeit zu erwerben und sich als Über-Mensch zu gerieren.

Geld wird Mittel zum ,transzendenten`Zweck, absolute Verfügungsgewalt über Menschen zu erlangen. Dupic rechnet damit, dass seine Klienten nicht zahlungsfähig sind, seine Schuldner werden (und Arbeitssklaven für seine Fabriken). Die Erniedrigung besteht darin, dass er ihnen vor Augen führt, dass sie unterlegen sind, weil sie Menschen sind, d. h. Wesen, die begehren. Er diktiert Elsa die vertragliche Bedingung, dass er ihr das Geld auf genau ein Jahr leihen wird. Die Wette mit der jungen Frau zielt darauf ab, ob es gelingt, auch sie zum „letzten Menschen“ (Nietzsche) zu erniedrigen.

„Bis zum 15. Oktober 1919. Wenn Sie mir an diesem Tag das Geld nicht zurückgeben können, werden Sie sich als von mir gekauft zu betrachten haben. Eigentlich hatte ich nicht mehr die Absicht, mir eine Geliebte zu nehmen; aber da sich eine so schöne Gelegenheit bietet. Sie sind ein zu hübsches Mädchen."18

Das ist eine Wette von beinahe faustischem Ausmaß, und der Böse geriert sich hier als einer, der vorgeblich das Gute will (Menschen wider alles kapitalistisches Kalkül Geld zinsfrei zu borgen) und gerade dadurch das Böse schafft. Nicht das primäre Begehren

17 Winder [Anm. 13], S. 131.

18 Winder [Anm. 13], S. 113. 
kommt hier - gegen alle bisherige Askese - zum Vorschein, sondern der Wunsch, das Mädchen zu überwältigen und zu erweisen, dass auch sie wie alle anderen Frauen nur ein Schattenbild der schamlosen Mutter ist. Gleichzeitig aber meldet sich bei Dupic eine Ambivalenz, ein sentimentaler Zug zu Wort: der heimliche Wunsch, dass Elsa doch anders sein könnte als die „letzten Menschen“. Zum einen stellen die Bedingungen, die er ihr diktiert, eine Überbietung des bösen Spiels der Macht dar, indem er aber zum anderen den Spieleinsatz derart erhöht, wird auch sein eigenes Ende absehbar. Er spielt buchstäblich sein Begehren von Elsa, das seine Macht bedroht, herunter, indem er den Spieleinsatz obszön erhöht. Die Schöne und das Biest. Elsa, die junge jüdische Frau, die sich in der bürgerlichen Welt etablieren will, wird zu einer prosaischen guten Fee. Das Biest zeigt seine Monstrosität auch in der Hoffnung, von ihr erlöst zu werden:

„Meine Brust ist über und über mit langen grauen Haaren bewachsen. Ich sehe aus wie ein Affe. " 19

Am Ende wird die Macht des Sekundär-Patriarchen gebrochen, für Elsa und Peter öffnet sich eine Welt danach. Es klingt so etwas wie ein gutes Ende an, das freilich sofort dementiert wird. Denn was Dupic hinterlässt, ist ein maßloser Streit unter seinen Erben.

\section{Quellen und Literatur}

Bachofen, Johann Jakob (1984): Mutterrecht und Urreligion (6. erweiterte Ausgabe). Stuttgart: Alfred Kröner.

Bauman, Zygmunt (1999): Das Unbehagen in der Postmoderne. Aus dem Englischen von Wiebke Schmaltz. Hamburg: Hamburger Ed.

Freud, Sigmund (1992): Das Ich und das Es. Metapsychologische Schriften. Einleitung von Alex Holder. Frankfurt a. M.: Fischer.

Hegel, Georg Wilhelm Friedrich (1970): Phänomenologie des Geistes. Werkausgabe, hrsg. von Eva Moldenhauer und Karl Markus Michel. Bd. 3. Frankfurt a. M.: Suhrkamp.

Musil, Robert (1978): Der Mann ohne Eigenschaften. Reinbek: Rowohlt.

Pfohlmann, Oliver (2016): Biografie. In: Nübel, Birgit - Wolf, Norbert Christian (hrsg.): Robert Musil Handbuch. Berlin - Boston: de Gruyter, S. 1-4.

Weinzierl, Ulrich (2014): Nachwort. Ein Gerechter unter den Schreibern. Ludwig Winder und sein „Thronfolger“. In: Winder, Ludwig: Der Thronfolger. Ein Franz-Ferdinand-Roman. Wien: Zsolnay, S. 553-572.

Winder, Ludwig (2014): Der Thronfolger. Ein Franz-Ferdinand-Roman. Wien: Zsolnay.

Winder, Ludwig (1987): Die nachgeholten Freuden [1927]. Wien: Zsolnay.

Wolf, Norbert Christian (2011): Kakanien als Gesellschaftskonstruktion. Robert Musils Sozioanalyse des 20. Jahrhunderts. Wien: Böhlau. 
Prof. Dr. Wolfgang Müller-Funk / wolfgang.mueller-funk@univie.ac.at

Institut für Europäische und Vergleichende Sprach- und Literaturwissenschaft, Universität Wien, Universitätsring 1, 1010 Wien, Österreich 